University of Nebraska - Lincoln

DigitalCommons@University of Nebraska - Lincoln

\title{
Swallowing Mechanics Associated With Artificial Airways, Bolus Properties, and Penetration-Aspiration Status in Trauma Patients
}

\author{
Angela M. Dietsch \\ University of Nebraska-Lincoln, angela.dietsch@unl.edu \\ Christopher B. Rowley \\ Medical College of Georgia, Augusta University \\ Nancy Pearl Solomon \\ Walter Reed National Military Medical Center, nancypsolomon@gmail.com \\ William G. Pearson Jr. \\ Medical College of Georgia, Augusta University, wpearson@augusta.edu
}

Follow this and additional works at: https://digitalcommons.unl.edu/specedfacpub

Part of the Special Education and Teaching Commons, Speech and Hearing Science Commons, and the Speech Pathology and Audiology Commons

Dietsch, Angela M.; Rowley, Christopher B.; Solomon, Nancy Pearl; and Pearson, William G. Jr.,

"Swallowing Mechanics Associated With Artificial Airways, Bolus Properties, and Penetration-Aspiration Status in Trauma Patients" (2017). Special Education and Communication Disorders Faculty Publications. 168.

https://digitalcommons.unl.edu/specedfacpub/168

This Article is brought to you for free and open access by the Department of Special Education and Communication Disorders at DigitalCommons@University of Nebraska - Lincoln. It has been accepted for inclusion in Special Education and Communication Disorders Faculty Publications by an authorized administrator of DigitalCommons@University of Nebraska - Lincoln. 


\title{
Swallowing Mechanics Associated With Artificial Airways, Bolus Properties, and Penetration-Aspiration Status in Trauma Patients
}

\author{
Angela M. Dietsch, ${ }^{\text {a,b }}$ Christopher B. Rowley, ${ }^{c}$ Nancy Pearl Solomon, ${ }^{\text {a }}$ and William G. Pearson, Jr. ${ }^{d}$
}

\begin{abstract}
Purpose: Artificial airway procedures such as intubation and tracheotomy are common in the treatment of traumatic injuries, and bolus modifications may be implemented to help manage swallowing disorders. This study assessed artificial airway status, bolus properties (volume and viscosity), and the occurrence of laryngeal penetration and/or aspiration in relation to mechanical features of swallowing.

Method: Coordinates of anatomical landmarks were extracted at minimum and maximum hyolaryngeal excursion from 228 videofluoroscopic swallowing studies representing 69 traumatically injured U.S. military service members with dysphagia. Morphometric canonical variate and regression analyses examined associations between swallowing mechanics and bolus properties based on artificial airway and penetration-aspiration status.
\end{abstract}

\begin{abstract}
Results: Significant differences in swallowing mechanics were detected between extubated versus tracheotomized $(D=1.32, p<.0001)$, extubated versus decannulated $(D=1.74$, $p<.0001)$, and decannulated versus tracheotomized ( $D=1.24$, $p<.0001$ ) groups per post hoc discriminant function analysis. Tracheotomy-in-situ and decannulated subgroups exhibited increased head/neck extension and posterior relocation of the larynx. Swallowing mechanics associated with (a) penetrationaspiration status and (b) bolus properties were moderately related for extubated and decannulated subgroups, but not the tracheotomized subgroup, per morphometric regression analysis. Conclusion: Specific differences in swallowing mechanics associated with artificial airway status and certain bolus properties may guide therapeutic intervention in traumabased dysphagia.
\end{abstract}

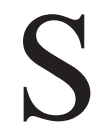
wallowing dysfunction is a common sequela of traumatic injury and particularly of the types of polytrauma often sustained by active-duty military service members (Solomon, Dietsch, Dietrich-Burns, Styrmisdottir, \& Armao, 2016). Even small disruptions to the timing, amplitude, or coordination of swallowing movements can lead to penetration or aspiration of a solid or liquid bolus into the airway (Arms, Dines, \& Tinstman, 1974), which is associated with a host of potentially serious

\footnotetext{
${ }^{\mathrm{a}}$ Walter Reed National Military Medical Center, National Military Audiology \& Speech Pathology Center, Bethesda, MD

${ }^{\mathrm{b}}$ University of Nebraska-Lincoln, Department of Special Education \& Communication Disorders

${ }^{\mathrm{c}}$ Medical College of Georgia, Augusta University

${ }^{\mathrm{d}}$ Department of Cellular Biology \& Anatomy, Medical College of

Georgia, Augusta University

Correspondence to Angela M. Dietsch: angela.dietsch@unl.edu

Editor: Julie Liss

Associate Editor: Catriona Steele

Received November 21, 2016

Revision received March 22, 2017

Accepted April 11, 2017

https://doi.org/10.1044/2017_JSLHR-S-16-0431
}

consequences. Individuals who aspirate are 11 times more likely to develop aspiration pneumonia compared with similar persons who do not aspirate (Martino et al., 2005). Swallowing impairment may also result in malnutrition, prolonged hospitalization, and increased mortality (Kozlow, Berenholtz, Garrett, Dorman, \& Pronovost, 2003; Macht et al., 2011). Because swallowing is a complex physiological process, there are many dependent and independent factors associated with swallowing impairment.

In trauma patients, primary sources of swallowing impairment often include sensorineural deficits, tissue damage, and higher-order neuronal dysfunction (Brown et al., 2011; Butler et al., 2011; Choi, Ryu, Kim, Kang, \& Yoo, 2011; Ding \& Logemann, 2005; Jung et al., 2012). Secondary factors, particularly the placement of an artificial airway (orotracheal intubation or tracheotomy) and deconditioning because of prolonged hospitalization, are common and may contribute to penetration-aspiration risk and swallow function (Bordon et al., 2011; Brown et al., 2011; Ding \& Logemann, 2005; Jung et al., 2012; Moraes, Sassi, Mangilli, Zilberstein, \& de Andrade, 2013; Rassameehiran, Klomjit,

Disclosure: The authors have declared that no competing interests existed at the time of publication. 


\section{Complimentary Author PDF: Not for Broad Dissemination}

Mankongpaisarnrung, \& Rakvit, 2015; Skoretz, Flowers, \& Martino, 2010; Skoretz, Yau, Ivanov, Granton, \& Martino, 2014; Solomon et al., 2016). Several studies suggest that duration of intubation positively correlates to incidence of swallowing dysfunction (Barker, Martino, Reichardt, Hickey, \& Ralph-Edwards, 2009; Bordon et al., 2011; Brodsky et al., 2014; Brown et al., 2011; DeVita \& Spierer-Rundback, 1990; Kwok, Davis, Cagle, Sue, \& Kaups, 2013; Skoretz et al., 2010, 2014) and aspiration frequency (Ajemian, Nirmul, Anderson, Zirlen, \& Kwasnik, 2001; Ceriana et al., 2015; El Solh, Okada, Bhat, \& Pietrantoni, 2003; Leder, Cohn, \& Moller, 1998). However, a paucity of kinematic data regarding the swallowing mechanics underlying the dysfunction associated with endotracheal intubation history leaves clinicians with little guidance about preliminary intervention targets in this population (Skoretz et al., 2014). The relationship between tracheotomy and swallow dysfunction is less clearly defined, with some studies showing an association between the presence of a tracheotomy tube and dysphagia/ aspiration (Ding \& Logemann, 2005; Elpern, Scott, Petro, \& Ries, 1994; Jung et al., 2012; Terk, Leder, \& Burrell, 2007; Tolep, Getch, \& Criner, 1996), whereas others find no link between tracheotomy and aspiration risk or swallowing kinematics (Kang, Choi, Yun, Kim, \& Ryu, 2012; Leder, Joe, Ross, Coelho, \& Mendes, 2005; Leder \& Ross, 2010; Sharma et al., 2007). In addition, a high incidence of silent aspiration in patients after extubation or tracheotomy suggests a relationship between artificial airway history and sensory integration, which could also influence cortical modulation of swallowing mechanics (Ding \& Logemann, 2005; Elpern et al., 1994; Leder et al., 1998). To summarize, findings in the extant literature regarding the relationship between artificial airway status and swallowing function are mixed, and a causal relationship has not been demonstrated. Initial injuries, medical interventions, and recovery factors all contribute to aspiration risk; unfortunately, they are not considerations that typically can be altered as part of dysphagia rehabilitation.

In contrast, aspiration risks that may be amenable to manipulation include some aspects of swallowing mechanics and bolus properties. Swallowing mechanics may be modified immediately with the use of swallowing maneuvers (such as an effortful swallow) or more sustainably with exercises (e.g., to improve hyolaryngeal excursion). These changes in swallowing mechanics, as represented by kinematic measurements, are correlated with shifts in aspiration risk (Choi et al., 2011; Molfenter \& Steele, 2014; Steele et al., 2011; Steele \& Cichero, 2014). However, these strategies may have limited effectiveness in the acute phases of recovery from trauma, when cognitive effects of injury and medication can interfere with implementation. In these situations, externally controlled compensations such as modified bolus properties may be particularly useful. Multiple investigations confirm that aspiration is more likely to occur with high-volume, low-viscosity boluses (Butler et al., 2010; Leder, Suiter, \& Green, 2011; Steele et al., 2015). Open questions remain as to whether aspiration risk is mitigated by the intrinsic bolus properties, such as bolus cohesiveness, or if there are differences in the swallowing mechanism's response to bolus properties that can promote safer swallowing in patients with an artificial airway.

Two recent developments provide means to explore questions regarding the effects of artificial airways and bolus property manipulation. First, a multidimensional database was compiled from the medical records of more than 200 U.S. military service members who sustained traumatic injuries resulting in dysphagia. A Bayesian Belief Network (BBN) analysis of the database revealed that intubation or tracheostomy prior to the first swallow evaluation were key variables in predicting swallowing outcomes at hospital discharge (Dietsch, Dietrich-Burns, \& Solomon, 2016; Solomon et al., 2016). This provides a foundation for further exploration of the effects of artificial airways on swallowing mechanics in this population. Second, advances in computational analysis of swallowing mechanics (CASM) provide a means to overcome unique challenges in discerning the effects of internal and external factors on the covariant muscle groups that displace the hyoid, larynx, pharynx, and tongue base. CASM enables multivariate analysis of these complex covariant elements in relation to multiple independent variables such as penetration-aspiration status, airway history, and bolus properties (Pearson, Taylor, Blair, \& Martin-Harris, 2016). Thus, CASM provides an extensive assessment of how pharyngeal swallowing mechanics and other variables interact to facilitate or undermine swallowing safety.

The present study examines swallow function in a cohort of U.S. military service members who sustained traumatic injuries resulting in dysphagia. On the basis of the $\mathrm{BBN}$ identification of artificial airway status as a primary associate of swallowing outcomes, CASM was used to assess the contributions of artificial airway status, bolus viscosity/volume, and penetration-aspiration status relative to swallowing mechanics. We hypothesized that swallowing mechanics would differ relative to airway history $\left(\mathrm{H}_{1}\right)$ and that mechanics associated with bolus properties would be predictive of mechanics associated with penetration-aspiration status $\left(\mathrm{H}_{2}\right)$, indicating that the swallowing mechanism responds to bolus types.

\section{Method \\ Participants}

Images from videofluoroscopic swallowing studies (VFSSs) and medical data were compiled as part of a larger Department of Defense-funded database project tracking the nature, management, and course of swallowing impairment as a result of traumatic injuries in military service members (Solomon et al., 2016). Participants were included in the database if they sustained combatrelated injuries for which they were admitted to Walter Reed Army Medical Center (WRAMC), National Naval Medical Center (NNMC), or Walter Reed National Military Medical Center (WRNMMC) between 2004 and 2014 (WRAMC and NNMC consolidated to form WRNMMC 


\section{Complimentary Author PDF: Not for Broad Dissemination}

in 2011), and if they were referred for evaluation of dysphagia during that hospitalization. Of 215 cases enrolled in the database, 69 participants with archival recordings of one or more VFSSs were included in the present report. CASM using VFSSs was performed in cooperation with Augusta University (AU). The institutional review boards at WRNMMC and AU approved the project, and informed consent was obtained for participants enrolled prospectively between 2012 and 2014. A waiver of consent was issued by the institutional review boards for cases enrolled retrospectively.

All participants were men, consistent with Department of Defense policies for combat-deployed military personnel during the data collection period. All had been intubated or tracheotomized at some point postinjury because of the nature of their injuries and/or for comfort during the long overseas transport from deployment regions to WRAMC/NNMC/WRNMMC. By the time of the VFSS, all previously intubated patients $(n=34)$ had been extubated. Of the tracheotomized patients $(n=35), 15$ still had the tracheotomy tube in place, 18 had been decannulated, and two had separate VFSSs conducted before and after decannulation. In most retrospectively included cases, it was not clear from the available medical records whether the tracheotomized persons had a cap or speaking valve in place at the time of the VFSS. The mean age of the study cohort at the time of admission was 29.1 years ( $S D$ age 7.6 years, range ages $19.7-43.1$ years).

\section{Procedures}

Researchers at WRNMMC reviewed medical records retrospectively to extract information for the database. During the course of clinical care, VFSSs conducted by speech-language pathologists (SLPs) and radiologists were recorded and archived. The attending clinicians determined the fluoroscopic settings and administered boluses, which may have included thin liquid, nectar, honey, pudding, and solid consistencies (Varibar product line; Bracco Imaging, Monroe Township, NJ) in varying amounts according to standard clinical procedures. Swallow trials were selected from these archived recordings for the purpose of biomechanical measurements.

Researchers trimmed the full-length VFSS recordings into deidentified clips of individual swallowing events in the lateral view (Ulead VideoStudio 11, Corel, Ottawa, Ontario, Canada). In all cases where they were available within the full-length VFSS recording for that participant and date, at least one thin liquid 5-ml trial and one pudding 5-ml trial were included. If more than one trial of a particular bolus type (e.g., of 5-ml thin liquid) was available within a single VFSS recording, the trial with the best image quality was selected for segmentation. Image quality was determined on the basis of the visibility of critical anatomical landmarks and contrast, head/body position, and the timing of fluoroscopy exposure to capture swallowing events (e.g., whether fluoroscopy captured the initial hyoid jump or the peak excursion of the hyoid). Additional swallow trials were included in the analysis if they were one step removed by either volume or consistency from the 5-ml thin liquid or pudding trials and also represented a change in penetration-aspiration status compared with the 5-ml thin liquid or pudding trial. For example, if the patient did not aspirate on the 5-ml trial of thin liquids but did aspirate on $10 \mathrm{ml}$ of thin liquid (the next volume increment in the facilities' standard bolus administration protocol), the 10-ml thin liquid trial was also included, whereas if the patient did not aspirate on either trial, only the 5-ml trial was included. In the same way, if laryngeal penetration was observed with 5-ml thin liquid but not with 5-ml nectar, the 5-ml nectar trial was also included in the analysis. With these criteria, a total of 228 clips were included for analysis, representing an average of 2.6 (range 1-6) clips per full-length VFSS.

A research SLP with more than 15 years of experience interpreting VFSSs (the first author) assigned PenetrationAspiration Scale (PAS; Rosenbek, Robbins, Roecker, Coyle, \& Wood, 1996) scores ranging from 1 (no airway compromise) to 8 (silent aspiration) for each swallowing event. Ten percent $(n=23)$ of the clips were randomly selected for rerating by the original rater and by another experienced research SLP (the third author). Intraclass correlation coefficients confirmed acceptable intra- and interrater reliability levels of .999 and .916, respectively. In subsequent analyses, these scores were reduced into two categories as follows: Scores of 1-2 were considered functionally normal, and scores of 3-8 indicated the occurrence of penetration and/or aspiration. In addition, each swallowing event was scored on the Modified Barium Swallow Impairment Scale (MBSImP; Martin-Harris et al., 2008) by two research SLPs certified in its use. Component 6 of the MBSImP, initiation of the pharyngeal swallow response (ranging from 0 [bolus head at ramus] to 4 [no initiation of the pharyngeal response]), served as a marker for sensory impairment because it is a fundamental reflection of the body's acknowledgment of the presence of a bolus. Component ratings of $0-1$ were operationally defined as functionally normal ( $n=153$ swallowing clips), whereas ratings of $2-$ 4 implied sensory impairment ( $n=75$ clips), reducing this variable to two levels.

A separate group of researchers, medical students at $\mathrm{AU}$, extracted the biomechanical data from the video clips after being trained and demonstrating reliability (interrater $r>.95$ for all coordinates when compared with an expert rater [the fourth author]). The raters were blinded to patient information and PAS scores. The coordinate mapping method used to document placement markers for this analysis has been described by Thompson et al. (2014). In brief, it involves review of each swallow trial using image-processing software applied to key frames within each swallow event (ImageJ; Rasband, 2012). By comparing anatomical landmarks at rest and maximum displacement as described below, biomechanical events are normalized within each participant.

Thompson et al. (2014) specified nine anatomical coordinates demarcating the mandible, nasal spine, three vertebrae, upper esophageal sphincter, posterior and anterior 


\section{Complimentary Author PDF: Not for Broad Dissemination}

aspects of the cricoid cartilage, and hyoid bone. In addition, this study included a 10th coordinate located at the pit of the valleculae to indicate the origin of the base of the tongue (Pearson et al., 2016). These 10 anatomical landmarks documented the action of muscle groups displacing the hyoid, larynx, tongue base, shortening of the pharynx, and posture of the head and neck (see Figure 1). To capture minimum swallowing excursion, all 10 coordinate markers were placed at the anatomical sites within the same preswallow frame. In each clip, this frame was selected from the start of the oral propulsive stage. For maximum excursion measures, markers were placed within the frame most relevant to the associated structure's movement. For example, in the frame that captured maximal hyoid excursion, Markers 1-5 (representing the skeletal frame of swallowing muscles) and 9 (representing the anterior horn of the hyoid bone) were moved to their new positions. Frames of maximum laryngeal elevation and upper esophageal sphincter opening were found individually, and the appropriate markers were moved to their new positions while verifying the location of Markers 1-5 to ensure stability of the patient's head position. For cases in which multiple swallows were required to clear the bolus, all measurements were taken from the first swallow. This process yielded two sets of 10 coordinates for each of the 228 swallowing clips.

The 228 available clips were categorized according to several parameters as shown in Table 1. In terms of artificial airway status, a tracheotomy tube was in situ during 43 clips and had previously been decannulated in 78 clips; the

Figure 1. Anatomical landmarks for computational analysis of swallowing mechanics. Ten anatomical landmarks are used to map muscle actions underlying hyoid movement (\#9), laryngeal elevation $(\# 7, \# 8)$, tongue base retraction (\#10 is placed at the inferior recess of the valleculae), pharyngeal shortening (\#6), and head and neck position (\#1-5). Coordinates representing these various elements of pharyngeal swallowing mechanics are annotated at minimum and maximum excursion of landmarks corresponding to oropharyngeal swallowing in prerecorded videofluoroscopic swallowing studies.

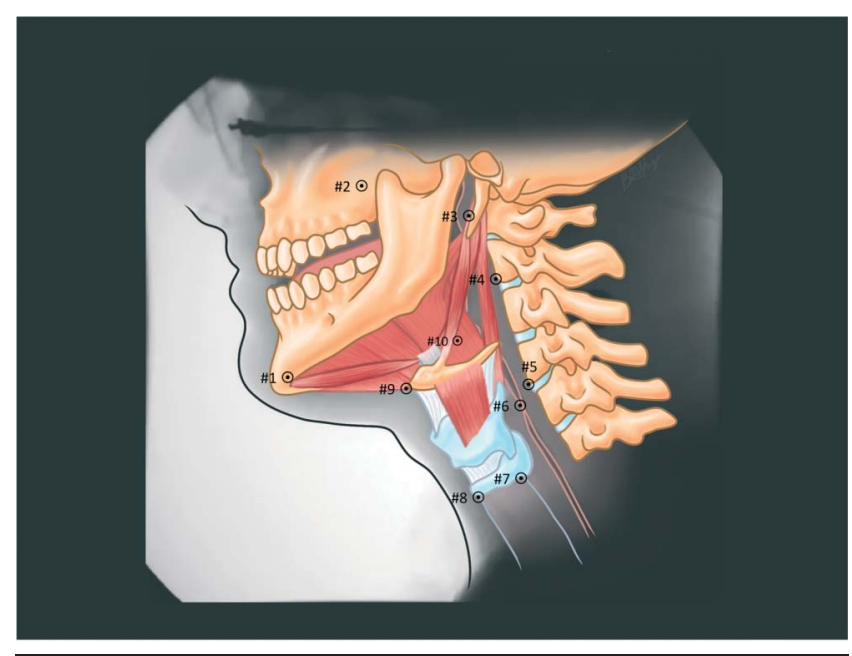

Table 1. Frequency table of categorical independent variables included in morphometric analysis.

\begin{tabular}{lr}
\hline Variable/Level & $\boldsymbol{n}$ \\
\hline $\begin{array}{l}\text { Swallowing excursion status of hyoid, larynx, pharynx, } \\
\quad \text { and tongue base }\end{array}$ \\
$\quad$ Minimum (at the beginning of the oral propulsion stage) & 228 \\
$\quad$ Maximum (peak excursion during pharyngeal stage) & 228 \\
Artificial airway status & 214 \\
$\quad$ Extubated & 86 \\
$\quad$ Decannulated & 156 \\
$\quad$ Tracheotomy in situ & 314 \\
Bolus volume & 142 \\
$\quad$ Small ( $\leq 5$ ml) & 254 \\
$\quad$ Large (> 5 ml) & 202 \\
Bolus viscosity & 260 \\
$\quad$ Low (thin liquid) & 196 \\
$\quad$ High (nectar, honey, pudding, or solid) \\
Penetration-aspiration status \\
$\quad$ Within functional limits (PAS 1-2) \\
$\quad$ Penetration-aspiration (PAS 3-8) \\
\hline Note. For each of 228 swallowing events from 72 videofluoroscopic \\
swallowing studies, two sets of coordinates were extracted (total \\
$N=556$ sets of coordinates). PAS = Penetration-Aspiration Scale \\
score.
\end{tabular}

107 remaining clips were from patients with a history of intubation but not tracheotomy associated with their traumatic injury. About two thirds of the 228 clips involved a small bolus $(n=157)$, and more than half used a thin liquid bolus $(n=127)$. The majority $(n=130)$ of swallow trials were within functional limits (PAS $=1,2)$, whereas the remainder $(n=98)$ showed evidence of laryngeal penetration or aspiration (PAS $=3-8)$.

\section{Analysis}

Coordinates and all categorical variables (excursion, penetration-aspiration status, airway history, bolus volume, and bolus viscosity) organized by Excel macros were imported into an integrated computational analytical software program (MorphoJ; Klingenberg, 2011). First, a Procrustes fit of 456 sets of coordinates was performed to control for participant size, head rotation, and image orientation (Klingenberg, 2011).

Canonical variate $(\mathrm{CV})$ analysis determined the relative contribution of each categorical variable on shape differences. No outliers in shape statistics were identified, which would have signaled possible coordinate mapping errors. The analysis identified that the first $\mathrm{CV}$, minimum to maximum excursion of swallowing (CV1), accounted for $66.02 \%$ of the morphological variance. The artificial airway group (CV2) comprised $8.61 \%$ of the variance. CV3, comprising $6.07 \%$ of the variance, was most highly associated with bolus volume, whereas CV4, comprising $5.64 \%$ of the variance, was most highly associated with bolus viscosity. CV5, comprising $3.81 \%$ of the variance, was identified with the presence/absence of penetrationaspiration. 


\section{Complimentary Author PDF: Not for Broad Dissemination}

Following this preliminary analysis to confirm airway history as a relevant covariate of swallowing mechanics, $\mathrm{H}_{1}$ was addressed through a series of post hoc discriminant function analyses (pairwise comparisons) to examine differences in mechanics across levels of artificial airway status for all 228 swallowing clips. For $\mathrm{H}_{2}$, a subset of swallow clips associated with boluses likely to represent high risk ( $>5 \mathrm{ml}$ of thin liquid viscosity) and low risk $(\leq 5 \mathrm{ml}$ of nectar viscosity or thicker) underwent morphometric regressions (similar to $r$ values and $r^{2}$ values) assessing mechanics associated with bolus properties as a predictor of penetration-aspiration mechanics. These regressions were performed for each airway cohort to control for the impact of airway history on morphology. For the extubated group, 23 high-risk boluses and 50 low-risk boluses were analyzed; 22 of these, all from the high-risk bolus group, showed evidence of penetration or aspiration. The tracheotomized group regression included 25 high-risk boluses and 28 lowrisk boluses with 26 normal swallows and 27 penetrationaspiration swallows. The decannulated group regression was composed of 15 high-risk boluses and 15-low risk boluses with 20 normal swallows and 10 penetration-aspiration swallows. Relationships among sensory impairment status, penetration-aspiration status, and airway history were assessed via three-way contingency tables with sensory integration and PAS status nested by airway history.

\section{Results}

The post hoc discriminant function analysis addressing $\mathrm{H}_{1}$ showed a significant difference between morphology associated with all airway status levels: extubated versus tracheotomized (Mahalanobis $D=1.32, p<.0001$ ), extubated versus decannulated $(D=1.74, p<.0001)$, and decannulated versus tracheotomized $(D=1.24, p<.0001)$. As indicated by the eigenvectors in Figure 2, the decannulated and tracheotomized groups demonstrated greater extension of the head and neck and more posterior displacement of the larynx during the swallow event compared with the extubated group, with decannulated group mechanics being more exaggerated. The tracheotomized group showed lowered hyolaryngeal position at both minimum and maximum excursion compared to the decannulated group (see Figure 2).

Regression analysis of penetration-aspiration status mechanics on bolus-risk mechanics within each airway cohort $\left(\mathrm{H}_{2}\right)$ resulted in a significant predictive relationship within the extubated group (see Figure 3A; regression coefficient $=-.44$, variance predicted $=16.96 \%, p<.0001$ ) and decannulated group (see Figure 3B; regression coefficient $=-.33$, variance predicted $=21.84 \%, p<.0001)$. The data indicated that high-risk bolus properties were predictive of penetration-aspiration in these groups. For the tracheotomized group, morphometric regression analysis showed that mechanics associated with bolus risk was not predictive of mechanics associated with penetrationaspiration status (see Figure 3C; regression coefficient $=$ .23 , variance predicted $=3.58 \%, p=.05)$. Here, the data
Figure 2. Difference in morphology by airway history. Superimposed eigenvectors at each anatomical landmark reflect the magnitude and direction of mean variance of the biomechanics of swallows for each artificial airway group. Tracheotomized and decannulated participants exhibited a more extended head and neck position and a more posterior laryngeal position compared with extubated participants as illustrated by the lines connecting skeletal landmarks for each airway cohort. Compared with tracheotomized patients, decannulated persons had decreased tongue base retraction, lower minimum hyoid position, and increased hyolaryngeal elevation, pharyngeal shortening, and head and neck extension.

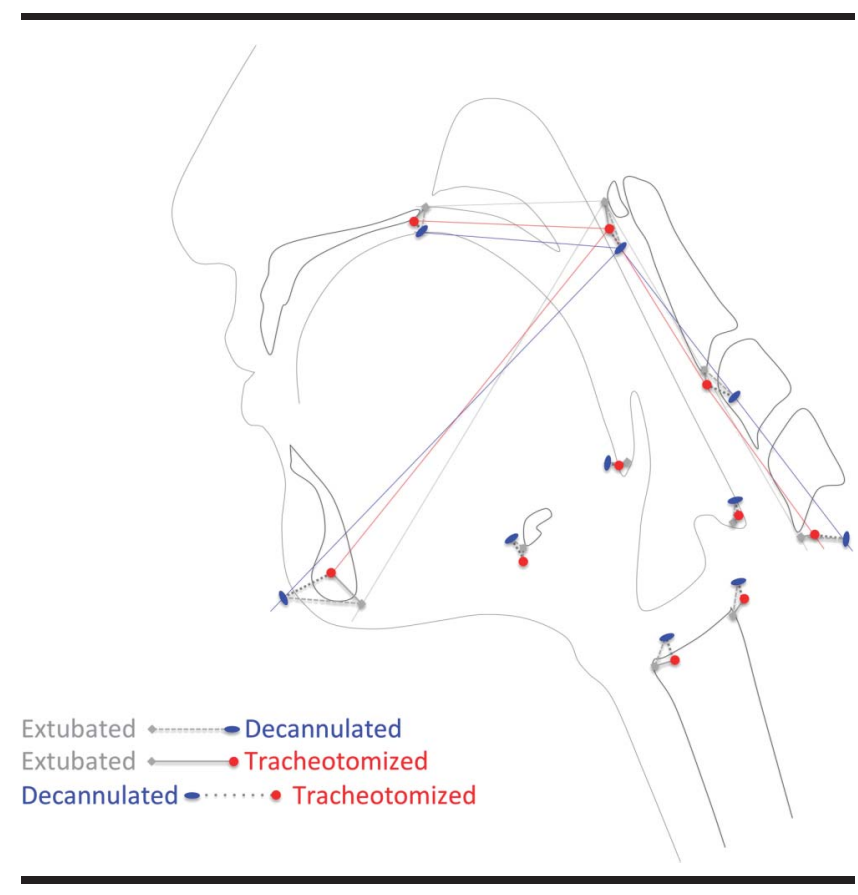

indicate a trend toward low-risk bolus properties predicting penetration-aspiration that did not reach statistical significance.

Contingency tables identified that when airway history was statistically controlled, swallows associated with sensory impairment were at greater risk for penetration-aspiration than those in which the timing of the pharyngeal response suggested intact sensorimotor integration $\left(G^{2}=17.12\right.$, $p<.0001)$. Neither the sensory impairment nor penetrationaspiration groups interacted significantly with airway history (sensory impairment, $G^{2}=2.58, p=.27$; penetrationaspiration, $G^{2}=2.52, p=.28$ ). Further post hoc testing using a Kruskal-Wallis test confirmed that there were no significant differences in pharyngeal timing response as measured by the MBSImP Component 6 score among airway history groups $(H=3.41, p=.18)$.

\section{Discussion}

In this cohort of traumatically injured patients, results of swallowing mechanics analysis generally supported study hypotheses. Airway history, identified as a primary associate of swallowing outcomes in a comprehensive database analysis of the same population (Dietsch et al., 


\section{Complimentary Author PDF: Not for Broad Dissemination}

Figure 3. Relationship between morphology associated with bolus risk and penetration-aspiration status for artificial airway history. Morphometric regression analysis indicates a predictive relationship between mechanics associated with high-risk bolus type (low viscosity, high volume) and mechanics associated with penetration-aspiration in the extubated (A) and decannulated (B) groups, respectively. In the tracheotomized group of swallows (C), morphometric regression analysis does not show a predictive relationship between bolus property risk and penetration-aspiration. WFL = within functional limits.
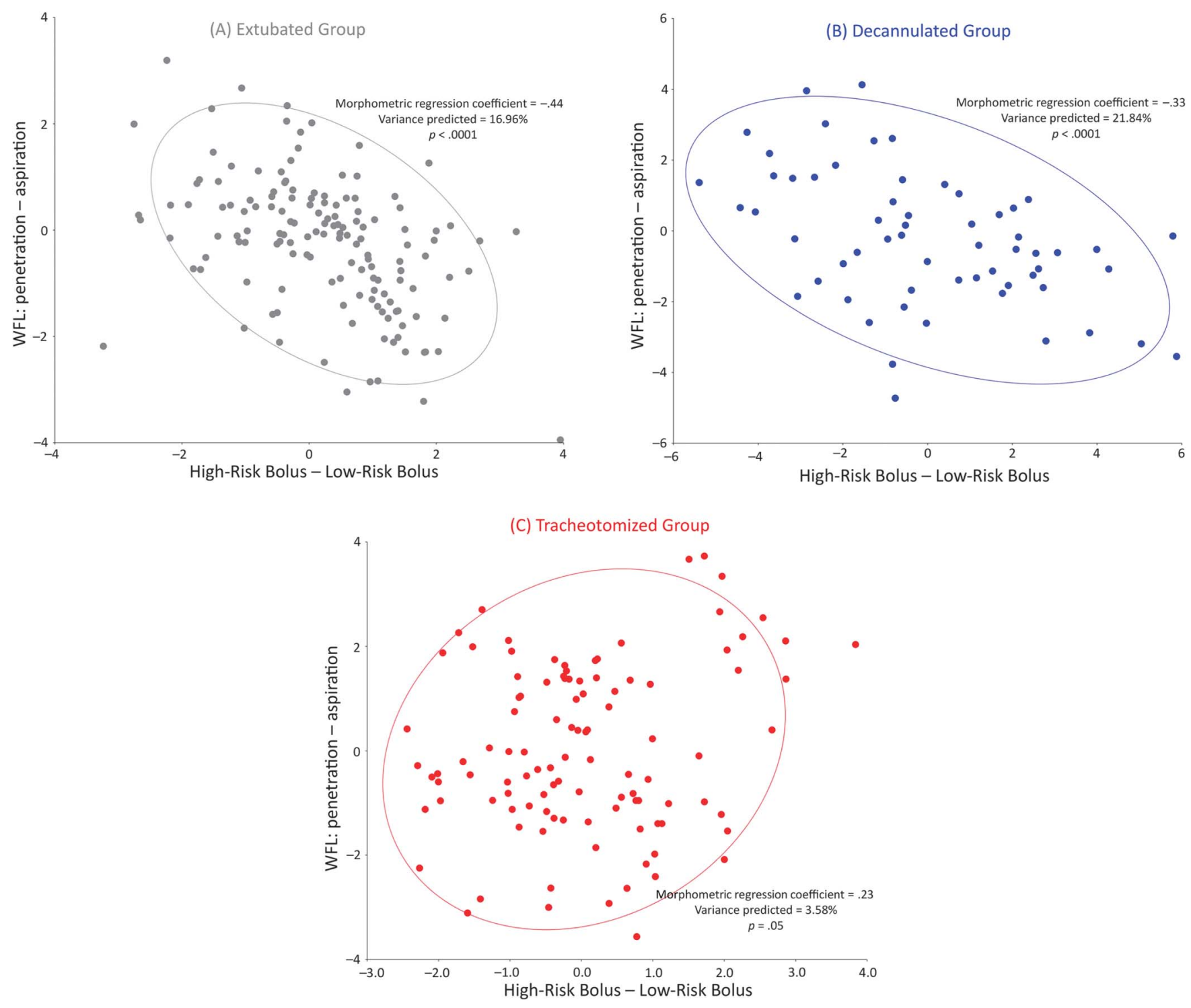

2016; Solomon et al., 2016), accounted for fundamental differences in swallowing mechanics across all three airway cohorts $\left(\mathrm{H}_{1}\right)$. One area of difference was specific to posterior laryngeal displacement and head and neck extension in previously or currently tracheotomized participants compared to extubated patients. Perhaps the combination of a tracheotomy tube and extended periods in a semireclined position for these acute-care patients resulted in neck extension and posterior displacement of the larynx that persisted even when the patients were seated upright for the VFSS. Another possible explanation stems from findings of a similar pattern of posterior laryngeal displacement in patients with impaired swallow-respiratory patterns (Martin-Harris et al., 2015; McFarland, Lund, \& Gagner, 1994; Tran, Martin-Harris, \& Pearson 2016). The tracheotomy tube (and the underlying respiratory compromise necessitating the tube) could contribute to altered swallow-respiratory coordination, for which the tracheotomized and decannulated patients spontaneously attempt to compensate via this altered laryngeal positioning. Previous literature exploring swallowing kinematics and tracheotomy does not document neck position during swallows (Ding \& Logemann, 2005; Jung et al., 2012; Terk et al., 2007) so it is unknown whether this could contribute to the disparities in reports regarding 


\section{Complimentary Author PDF: Not for Broad Dissemination}

tracheotomy effects on swallowing movements, safety, and functional outcomes. A study of tracheotomized patients, including respiratory-swallow phase data, would be helpful to address this question.

Airway-related differences in swallowing physiology extended to the bolus-related variables considered in this analysis, generally supporting $\mathrm{H}_{2}$. Swallowing mechanics associated with high-risk bolus properties (large volume, low viscosity) were predictive of swallowing mechanics associated with penetration-aspiration in extubated and decannulated groups, but not in the tracheotomized group. These findings support and extend the literature suggesting that aspiration risk can be mitigated by manipulating bolus viscosity and/or volume in some patients. Previous VFSS findings in patients with dysphagia identified reductions in prevalence and risk of aspiration with thickerconsistency boluses as compared with thin liquid barium (Clavé et al., 2006; Kuhlemeier, Palmer, \& Rosenberg, 2001; Leonard, White, McKenzie, \& Belafsky, 2014; Rofes, Arreola, Mukherjee, Swanson, \& Clavé, 2014). Moreover, the frequency of aspiration events as documented via fiberoptic endoscopic swallowing evaluations in healthy older adults was lower at smaller $(5-10 \mathrm{ml})$ bolus volumes compared with larger $(20 \mathrm{ml})$ volumes (Butler et al., 2010). Limited evidence regarding changes in motor components of the oral phase of swallowing (specifically tongue-to-palate pressure) in response to bolus consistency has been mixed (Steele, Molfenter, Peladeau-Pigeon, Polacco, \& Yee, 2014; Vickers et al., 2015), leaving questions as to whether aspiration risk is mitigated simply by altered fluid dynamics or a more complex interaction with swallowing physiology. The current results directly address this gap by providing quantitative evidence of mechanical changes in pharyngeal phase response with boluses of varying properties in the extubated and decannulated groups. The predictive relationship between bolus-properties mechanics and penetrationaspiration mechanics suggests that aspiration risk was influenced not just by factors related to bolus flow and cohesion, but also by sensorimotor responses to bolus properties. This was confirmed by the strong relationship between sensory impairment status and penetration-aspiration status that was not associated with a particular airway cohort. These results provide further preliminary evidence for bolusproperty manipulation as a neurorehabilitative intervention for dysphagia in addition to a compensatory strategy to prevent aspiration on a particular swallow event.

The tracheotomized cohort differed from the other two groups in multiple ways relative to the study hypotheses. First, the presence of a tracheotomy tube was associated with a lower hyolaryngeal position compared with decannulated and extubated participants. This agrees with several kinematic analyses of swallow function across multiple tracheotomy conditions (e.g., immediately before or after placement or decannulation, cuff inflated/deflated) describing reductions in hyolaryngeal excursion associated with tracheotomy (Ding \& Logemann, 2005; Jung et al., 2012; Terk et al., 2007). Second, this group's motor response to high-risk versus low-risk bolus properties did not achieve statistical significance in predicting mechanics underlying penetration-aspiration status in the tracheotomized group, and the overall relationship sloped in the opposite direction as that of the other two groups (see Figures $3 \mathrm{~A}-\mathrm{C}$ ). The tracheotomized group did not respond to the bolus properties in an effective compensatory way in terms of swallowing mechanics. Third, swallows from the tracheotomized group had a similar risk of being linked to sensory impairments as did those from the extubated group, but were more likely to exhibit concurrent sensory impairments and penetration-aspiration than swallows from either of the other airway groups. It appears that the tube alters the body's capacity to respond to bolus properties because of physical limitations on the degrees of freedom of movement available, sensorimotor integration issues, or both. Taken together, these results suggest that the need for and/or the presence of the tracheotomy tube alters swallowing mechanics in ways that are not present after decannulation. To be clear, this does not indicate that aspiration risk is necessarily increased by the presence of a tracheotomy tube or that all such patients would benefit from bolus modifications. Instead, it suggests that for persons with a tracheotomy, the primary potential value of altered bolus properties may be as a compensatory strategy specific to the flow and cohesion properties of that bolus. Extubated and decannulated persons may benefit similarly from modified bolus flow/cohesion as a compensatory strategy, and they may gain an additional rehabilitative advantage from improved swallowing mechanics in response to the altered bolus properties $\left(\mathrm{H}_{2}\right)$.

Additional exploratory analyses of study data revealed secondary findings that may be unique to this study cohort. For example, all artificial airway groups exhibited relatively typical patterns of swallowing mechanics (hyoid movement, laryngeal elevation, pharyngeal shortening, and tongue base retraction) from minimum to maximum excursion. This indicates that even the tracheotomized patients in this cohort were able to overcome any effects of the tube to achieve near-normal swallowing physiology. Given their status as active-duty military service members, this young, superfit, and otherwise healthy study sample may have more reserve to overcome traumatic injury and artificial airway effects on swallowing than would be present in a more heterogeneous civilian cohort. Also, study participants were drawn from a limited number of military hospitals with similar standard operating procedures; these clinical protocols may dictate that VFSSs occur at different (and possibly more consistent) points in recovery than would occur in a civilian health care setting.

Another unexpected exploratory finding was that post hoc regression analysis showed no predictive relationship between artificial airway status and penetration-aspiration (regression coefficient $=.01$, variance predicted $=0.05 \%$, $p=.64)$. This result further underscores that despite airwayrelated differences in swallowing mechanics, the functional and clinical impacts of those differences were negligible in this limited sample. Instead, the penetration-aspiration events were linked to generalized reductions in all swallowing 
movements regardless of airway history. Although an individualized treatment plan based on instrumental swallowing assessment and patient-specific morphometric data is always preferable, the clinical reality is that in some cases or settings, preliminary therapy is initiated while an instrumental evaluation is still pending. In such situations, global exercises, such as effortful nonbolus swallows, best match the pattern of generalized deficits in swallowing mechanics observed here and may be the most appropriate approach until the swallow can be visualized instrumentally and a more customized plan can be developed.

The nature of this largely retrospective analysis prevented the inclusion of some factors that could be relevant to swallowing mechanics and should be explored in future work. Virtually all traumatically injured patients in this cohort were injured in deployment zones, and they were sedated and intubated to maintain comfort during the long overseas flight stateside. This precluded the inclusion of a cohort of nonintubated/nontracheotomized patients with similar injuries, ages, and premorbid fitness levels. Because documentation frequently did not capture whether a speaking valve or a tracheotomy cap was present during VFSS, the present analysis does not account for any effects of this on swallowing mechanics. As is the case with most civilian trauma patients without massive facial trauma, most patients who were eventually tracheotomized also had been intubated for some initial period of time, and this was not included as a distinct variable. Some participants had traumatic brain injuries, some had maxillofacial trauma (MFT), and some had both or neither of these issues as part of their injury profile. An analysis of the larger database cohort revealed that MFT was associated with earlier referral for swallow evaluation, greater initial swallowing impairments, and more prolonged dysphagia than those without MFT, whereas brain injury was not associated with statistically significant differences on these non-CASM measures (Dietsch et al., 2016). Factors such as duration of artificial airway placement, timing between extubation/decannulation/ tracheotomy and VFSS, and type/size of tracheotomy tube were determined by clinical providers and varied from participant to participant. In addition, the young and highly fit service members in this analysis are not necessarily representative of typical trauma patients in civilian hospitals. Advanced age is independently associated with disadvantageous changes in biomechanical features of swallowing (Leonard, Kendall, \& McKenzie, 2004; Logemann et al., 2000; Logemann, Pauloski, Rademaker, \& Kahrilas, 2002) and an increased risk of aspiration in persons who have previously been intubated (Bordon et al., 2011; Kwok et al., 2013; Skoretz et al., 2014). In addition, hand strength is positively correlated to swallowing function (Butler et al., 2011; Hathaway et al., 2015), further supporting that the cohort included in this study may have more capacity to compensate for the effects of injury, artificial airways, and bolus manipulations than more aged or frail individuals. Nonetheless, CASM was useful for identifying significant differences in swallowing biomechanics associated with airway history groups. A larger data set may allow for more precise understanding of the impact of these additional factors on pharyngeal mechanics using CASM in future studies.

The results of the current investigation help clarify and expand the pool of knowledge regarding pharyngeal swallowing mechanics associated with artificial airway history, bolus properties, and penetration-aspiration status in traumatically injured patients. Tracheotomy affects swallowing mechanics, although it is unclear whether adaptations in laryngeal posture and head/neck extension are maladaptive or compensatory. Because post hoc analyses failed to show whether these airway-related differences were predictive of penetration-aspiration status, these results can suggest only preliminary therapeutic targets until a patient is able to participate in instrumental assessment for a more individualized management plan. Furthermore, results suggest that for some populations, bolus properties may contribute to adverse or beneficial changes in swallowing mechanics beyond immediate mitigation of aspiration. As such, bolus modifications may offer both compensatory and rehabilitative potential for some patients with dysphagia by improving bolus flow/cohesion contributions and sensorimotor responses. Quantification of the effects of injury and intervention variables on swallowing will be enhanced by the use of CASM to analyze and visualize multiple elements of pharyngeal swallowing mechanics captured in VFSS and promises to further refine dysphagia management in a range of clinical populations.

\section{Acknowledgments}

This research was funded by the United States Army Medical Research and Development Program (W81XWH-12-2-0021; PI: Solomon). The authors sincerely thank Katie Dietrich-Burns, Cody Hightower, and L. Kevin Heard for contributions to data extraction and measurement.

The views expressed in this presentation are those of the authors and do not reflect official policies of the U.S. Department of Defense or the U.S. Government. The identification of specific products or scientific instrumentation does not constitute endorsement, implied endorsement, or preferential treatment on the part of the authors, the Department of Defense, or any component agency.

\section{References}

Ajemian, M. S., Nirmul, G. B., Anderson, M. T., Zirlen, D. M., \& Kwasnik, E. M. (2001). Routine fiberoptic endoscopic evaluation of swallowing following prolonged intubation: Implications for management. Archives of Surgery, 136, 434- 437.

Arms, R. A., Dines, D. E., \& Tinstman, T. C. (1974). Aspiration pneumonia. Chest, 65, 136-139.

Barker, J., Martino, R., Reichardt, B., Hickey, E. J., \& RalphEdwards, A. (2009). Incidence and impact of dysphagia in patients receiving prolonged endotracheal intubation after cardiac surgery. Canadian Journal of Surgery, 52, 119-124.

Bordon, A., Bokhari, R., Sperry, J., Testa, D., IV, Feinstein, A., \& Ghaemmaghami, V. (2011). Swallowing dysfunction after prolonged intubation: Analysis of risk factors in trauma patients. 
The American Journal of Surgery, 202, 679-683. https://doi. org/10.1016/j.amjsurg.2011.06.030

Brodsky, M. B., Gellar, J. E., Dinglas, V. D., Colantuoni, E., Mendez-Tellez, P. A., Shanholtz, C., ... Needham, D. M. (2014). Duration of oral endotracheal intubation is associated with dysphagia symptoms in acute lung injury patients. Journal of Critical Care, 29, 574-579. https://doi.org/10.1016/j.jcrc.2014. 02.015

Brown, C. V., Hejl, K., Mandaville, A. D., Chaney, P. E., Stevenson, G., \& Smith, C. (2011). Swallowing dysfunction after mechanical ventilation in trauma patients. Journal of Critical Care, 26(1), 108.e9-e13. https://doi.org/10.1016/j.jcrc.2010.05.036

Butler, S. G., Stuart, A., Leng, X., Rees, C., Williamson, J., \& Kritchevsky, S. B. (2010). Factors influencing aspiration during swallowing in healthy older adults. The Laryngoscope, 120, 2147-2152. https://doi.org/10.1002/lary.21116

Butler, S. G., Stuart, A., Leng, X., Wilhelm, E., Rees, C., Williamson, J., \& Kritchevsky, S. B. (2011). The relationship of aspiration status with tongue and handgrip strength in healthy older adults. The Journals of Gerontology, Series A: Biological Sciences and Medical Sciences, 66, 452-458. https://doi.org/ 10.1093/gerona/glq234

Ceriana, P., Carlucci, A., Schreiber, A., Fracchia, C., Cazzani, C., Dichiarante, M., ... Nava, S. (2015). Changes of swallowing function after tracheostomy: A videofluoroscopy study. Minerva Anestesiologica, 81, 389-397.

Choi, K. H., Ryu, J. S., Kim, M. Y., Kang, J. Y., \& Yoo, S. D. (2011). Kinematic analysis of dysphagia:Significant parameters of aspiration related to bolus viscosity. Dysphagia, 26, 392-398. https://doi.org/10.1007/s00455-011-9325-5

Clavé, P., de Kraa, M., Arreola, V., Girvent, M., Farré, R., Palomera, E., \& Serra-Prat, M. (2006). The effect of bolus viscosity on swallowing function in neurogenic dysphagia. Alimentary Pharmacology and Therapeutics, 24, 1385-1394. https://oi.org/ 10.1111/j.1365-2036.2006.03118.x

DeVita, M. A., \& Spierer-Rundback, L. (1990). Swallowing disorders in patients with prolonged orotracheal intubation or tracheostomy tubes. Critical Care Medicine, 18, 1328-1330.

Dietsch, A. M., Dietrich-Burns, K. E., \& Solomon, N. P. (2016). Dysphagia outcomes after combat-related maxillofacial traumatic injuries [Abstract]. Dysphagia, 31, 816. https://doi.org/ 10.1007/s00455-016-9752-4

Ding, R., \& Logemann, J. A. (2005). Swallow physiology in patients with trach cuff inflated or deflated: A retrospective study. Head \& Neck, 27, 816. https://doi.org/10.1002/hed.20248

Elpern, E. H., Scott, M. G., Petro, L., \& Ries, M. H. (1994). Pulmonary aspiration in mechanically ventilated patients with tracheostomies. Chest, 105, 563-566.

El Solh, A., Okada, M., Bhat, A., \& Pietrantoni, C. (2003). Swallowing disorders post orotracheal intubation in the elderly. Intensive Care Medicine, 29, 1451-1455. https://doi.org/10.1007/ s00134-003-1870-4

Hathaway, B., Baumann, B., Byers, S., Wasserman-Wincko, T., Badhwar, V., \& Johnson, J. (2015). Handgrip strength and dysphagia assessment following cardiac surgery. The Laryngoscope, 125, 2330-2332. https://doi.org/10.1002/ lary. 25175

Jung, S. J., Kim, D. Y., Kim, Y. W., Koh, Y. W., Joo, S. Y., \& Kim, E. S. (2012). Effect of decannulation on pharyngeal and laryngeal movement in post-stroke tracheostomized patients. Annals of Rehabilitation Medicine, 36, 356-364. https://doi.org/ 10.5535/arm.2012.36.3.356

Kang, J. Y., Choi, K. H., Yun, G. J., Kim, M. Y., \& Ryu, J. S. (2012). Does removal of tracheostomy affect dysphagia? A kinematic analysis. Dysphagia, 27, 498-503. https://doi.org/ 10.1007/s00455-012-9396-y

Klingenberg, C. P. (2011). MorphoJ: An integrated software package for geometric morphometrics. Molecular Ecology Resources, 11, 353-357. https://doi.org/10.1111/j.1755-0998.2010. 02924.x

Kozlow, J. H., Berenholtz, S. M., Garrett, E., Dorman, T., \& Pronovost, P. J. (2003). Epidemiology and impact of aspiration pneumonia in patients undergoing surgery in Maryland, 1999-2000. Critical Care Medicine, 31, 1930-1937. https://doi. org/10.1097/01.CCM.0000069738.73602.5F

Kuhlemeier, K. V., Palmer, J. B., \& Rosenberg, D. (2001). Effect of liquid bolus consistency and delivery method on aspiration and pharyngeal retention in dysphagia patients. Dysphagia, 16, 119-122.

Kwok, A. M., Davis, J. W., Cagle, K. M., Sue, L. P., \& Kaups, K. L. (2013). Post-extubation dysphagia in trauma patients: It's hard to swallow. The American Journal of Surgery, 206, 924-927; discussion 927-928. https://doi.org/10.1016/j.amjsurg. 2013.08.010

Leder, S. B., Cohn, S. M., \& Moller, B. A. (1998). Fiberoptic endoscopic documentation of the high incidence of aspiration following extubation in critically ill trauma patients. Dysphagia, 13, 208-212.

Leder, S. B., Joe, J. K., Ross, D. A., Coelho, D. H., \& Mendes, J. (2005). Presence of a tracheotomy tube and aspiration status in early, postsurgical head and neck cancer patients. Head \& Neck, 27, 757-761. https://doi.org/10.1002/hed.20239

Leder, S. B., \& Ross, D. A. (2010). Confirmation of no causal relationship between tracheotomy and aspiration status: A direct replication study. Dysphagia, 25, 35-39. https://doi.org/ 10.1007/s00455-009-9226-Z

Leder, S. B., Suiter, D. M., \& Green, B. G. (2011). Silent aspiration risk is volume-dependent. Dysphagia, 26, 304-309. https:// doi.org/10.1007/s00455-010-9312-2

Leonard, R., Kendall, K. A., \& McKenzie, S. (2004). Structural displacements affecting pharyngeal constriction in nondysphagic elderly and nonelderly adults. Dysphagia, 19, 133-141.

Leonard, R. J., White, C., McKenzie, S., \& Belafsky, P. C. (2014). Effects of bolus rheology on aspiration in patients with dysphagia. Journal of the Academy of Nutrition and Dietetics, 114, 590-594. https://doi.org/10.1016/j.jand.2013. 07.037

Logemann, J. A., Pauloski, B. R., Rademaker, A. W., Colangelo, L. A., Kahrilas, P. J., \& Smith, C. H. (2000). Temporal and biomechanical characteristics of oropharyngeal swallow in younger and older men. Journal of Speech, Language, and Hearing Research, 43, 1264-1274.

Logemann, J. A., Pauloski, B., Rademaker, A. W., \& Kahrilas, P. J. (2002). Oropharyngeal swallow in younger and older women: Videofluoroscopic analysis. Journal of Speech, Language, and Hearing Research, 45, 434445.

Macht, M., Wimbish, T., Clark, B. J., Benson, A. B., Burnham, E. L., Williams, A., \& Moss, M. (2011). Postextubation dysphagia is persistent and associated with poor outcomes in survivors of critical illness. Critical Care, 15(5), R231. https:// doi.org/10.1186/cc10472

Martino, R., Foley, N., Bhogal, S., Diamant, N., Speechley, M., \& Teasell, R. (2005). Dysphagia after stroke: Incidence, diagnosis, and pulmonary complications. Stroke, 36, 2756-2763. https://doi.org/10.1161/01.STR.0000190056.76543.eb

Martin-Harris, B., Brodsky, M. B., Michel, Y., Castell, D. O., Schleicher, M., Sandidge, J., ... Blair, J. (2008). MBS measurement tool for swallow impairment-MBSImP: Establishing 


\section{Complimentary Author PDF: Not for Broad Dissemination}

a standard. Dysphagia, 23, 392-405. https://doi.org/10.1007/ s00455-008-9185-9

Martin-Harris, B., McFarland, D., Hill, E. G., Strange, C. B., Focht, K. L., Wan, Z., ... McGrattan, K. (2015). Respiratoryswallow training in patients with head and neck cancer. Archives of Physical Medicine and Rehabilitation, 96, 885-893.

McFarland, D. H., Lund, J. P., \& Gagner, M. (1994). Effects of posture on the coordination of respiration and swallowing. Journal of Neurophysiology, 72, 2431-2437.

Molfenter, S. M., \& Steele, C. M. (2014). Kinematic and temporal factors associated with penetration-aspiration in swallowing liquids. Dysphagia, 29, 269-276. https://doi.org/10.1007/ s00455-013-9506-5

Moraes, D. P., Sassi, F. C., Mangilli, L. D., Zilberstein, B., \& de Andrade, C. R. (2013). Clinical prognostic indicators of dysphagia following prolonged orotracheal intubation in ICU patients. Critical Care, 17(5), R243. https://doi.org/10.1186/cc13069

Pearson, W. G., Jr., Taylor, B. K., Blair, J., \& Martin-Harris, B. (2016). Computational analysis of swallowing mechanics underlying impaired epiglottic inversion. The Laryngoscope, 126, 1854-1858. https://doi.org/10.1002/lary.25788

Rasband, W. (2012). ImageJ. Bethesda, MD: National Institutes of Health.

Rassameehiran, S., Klomjit, S., Mankongpaisarnrung, C., \& Rakvit, A. (2015). Postextubation dysphagia. Proceedings (Baylor University Medical Center), 28(1), 18-20.

Rofes, L., Arreola, V., Mukherjee, R., Swanson, J., \& Clavé, P. (2014). The effects of a xanthan gum-based thickener on the swallowing function of patients with dysphagia. Alimentary Pharmacology and Therapeutics, 39, 1169-1179. https://doi. org/10.1111/apt.12696

Rosenbek, J. C., Robbins, J. A., Roecker, E. B., Coyle, J. L., \& Wood, J. L. (1996). A Penetration-Aspiration Scale. Dysphagia, 11, 93-98.

Sharma, O. P., Oswanski, M. F., Singer, D., Buckley, B., Courtright, B., Raj, S. S., ... Gandaio, A. (2007). Swallowing disorders in trauma patients: Impact of tracheostomy. The American Surgeon, 73, 1117-1121.

Skoretz, S. A., Flowers, H. L., \& Martino, R. (2010). The incidence of dysphagia following endotracheal intubation: A systematic review. Chest, 137, 665-673. https://doi.org/10.1378/ chest.09-1823

Skoretz, S. A., Yau, T. M., Ivanov, J., Granton, J. T., \& Martino, R. (2014). Dysphagia and associated risk factors following extubation in cardiovascular surgical patients. Dysphagia, 29, 647-654. https://doi.org/10.1007/s00455-014-9555-4
Solomon, N. P., Dietsch, A. M., Dietrich-Burns, K. E., Styrmisdottir, E. L., \& Armao, C. S. (2016). Dysphagia management and research in an acute-care military treatment facility: The role of applied informatics. Military Medicine, 181(5. Suppl), 138-144. https://doi.org/10.7205/MILMED-D-15-00170

Steele, C. M., Alsanei, W. A., Ayanikalath, S., Barbon, C. E., Chen, J., Cichero, J. A., ... Wang, H. (2015). The influence of food texture and liquid consistency modification on swallowing physiology and function: A systematic review. Dysphagia, 30, 2-26. https://doi.org/10.1007/s00455-014-9578-x

Steele, C. M., Bailey, G. L., Chau, T., Molfenter, S. M., Oshalla, M., Waito, A. A., \& Zoratto, D. C. (2011). The relationship between hyoid and laryngeal displacement and swallowing impairment. Clinical Otolaryngology, 36, 30-36. https://doi.org/ 10.1111/j.1749-4486.2010.02219.x

Steele, C. M., \& Cichero, J. A. (2014). Physiological factors related to aspiration risk: A systematic review. Dysphagia, 29, 295-304. https://doi.org/10.1007/s00455-014-9516-y

Steele, C. M., Molfenter, S. M., Peladeau-Pigeon, M., Polacco, R. C., \& Yee, C. (2014). Variations in tongue-palate swallowing pressures when swallowing xanthan gum-thickened liquids. Dysphagia, 29, 678-684. https://doi.org/10.1007/s00455-0149561-6

Terk, A. R., Leder, S. B., \& Burrell, M. I. (2007). Hyoid bone and laryngeal movement dependent upon presence of a tracheotomy tube. Dysphagia, 22, 89-93. https://doi.org/10.1007/ s00455-006-9057-0

Thompson, T. Z., Obeidin, F., Davidoff, A. A., Hightower, C. L., Johnson, C. Z., Rice, S. L., .. Pearson, W. G., Jr. (2014). Coordinate mapping of hyolaryngeal mechanics in swallowing. Journal of Visualized Experiments, (87). https://doi.org/ $10.3791 / 51476$

Tolep, K., Getch, C. L., \& Criner, G. J. (1996). Swallowing dysfunction in patients receiving prolonged mechanical ventilation. Chest, 109, 167-172.

Tran, T. T. A., Martin-Harris, B., \& Pearson, W. G., Jr. (2016). Improvements resulting from respiratory-swallow phase training visualized in patient-specific computational analysis of swallowing mechanics. Computer Methods in Biomechanics and Biomedical Engineering: Imaging \& Visualization, 1-7. https://doi.org/10.1080/21681163.2016.1152567

Vickers, Z., Damodhar, H., Grummer, C., Mendenhall, H., Banaszynski, K., Hartel, R., ... Robbins, J. (2015). Relationships among rheological, sensory texture, and swallowing pressure measurements of hydrocolloid-thickened fluids. Dysphagia, 30, 702-713. https://doi.org/10.1007/s00455-015-9647-9 